\title{
RESULTS OF THE BETA SCORPII OCCULTATION BY JUPITER ON MAY 13, 1971
}

\author{
L. VAPILLON \\ Groupe Planètes, Observatoire de Paris-Meudon, France
}

\begin{abstract}
The aim of this paper is to present results of temperature and density profiles of the upper atmosphere of Jupiter as deduced from observation of the occultation of $\beta$ Sco on May 13, 1971.

The occultation light curve of $\beta$ Sco is shown. The analysis of data is based on the Abelian integral inversion. Jovian refractivity profiles and typical temperature and density profiles are also shown.

The extent of the atmospheric zone where results are significant is discussed.

It is shown that no valid information about the thermosphere has been obtained.
\end{abstract}

\section{Introduction}

The interest in the occultation of a star by a planet is that it enables one to investigate the atmosphere of the planet. It is known that the gradual drop in the flux from the star during the occultation is due to differential refraction in progressively denser (and, therefore, deeper) layers of the planet's atmosphere. Clearly, it is desirable to be able to observe the star for as long as possible during the occultation, which requires the capacity of making precise measurements of low stellar fluxes. One must, therefore, have access to a large telescope. Our observations of the occultation of $\beta$ Sco by Jupiter on May 13, 1971, were made with the $2 \mathrm{~m}$ telescope of the Radcliffe Observatory, Pretoria. The observed flux of $\beta$ Sco fell to $1 / 10$ of its initial value over a period of about a minute, and to $1 / 100$ of its initial value in $3 \mathrm{~min}$. The measurement of these very low values of the stellar flux are all the more difficult close to the light limb of the planet.

The latter difficulty may be overcome by using a photometer equipped with a diaphragm of suitable diameter the outer parts of which reflect the light from the limb of the planet to an auxiliary photomultiplier. From the variations in the flux received by this photomultiplier, one may correct for pointing errors, turbulence etc... even a posteriori.

In order to increase the contrast of the star relative to the planet, the observations were made with an interference filter, of $9 \AA$ width, centred on the wavelength of the Ca K line (3934 $\AA$ ). By this means, we were able to observe $\beta$ Sco for over $900 \mathrm{~s}$ at immersion and $820 \mathrm{~s}$ at emersion, (Figures $1 \mathrm{a}$ and $1 \mathrm{~b}$ ).

The light curve which we obtained shows that a substantial error is committed if the observations stop too soon or if too small a telescope is used. It is evident from the light curve that only after a period of about $5 \mathrm{~min}$ does the flux of the star become truly indistinguishable from the background noise. If the observations stop before this point is reached, the zero of the light curve is placed too high, with the consequences that will be discussed below. 


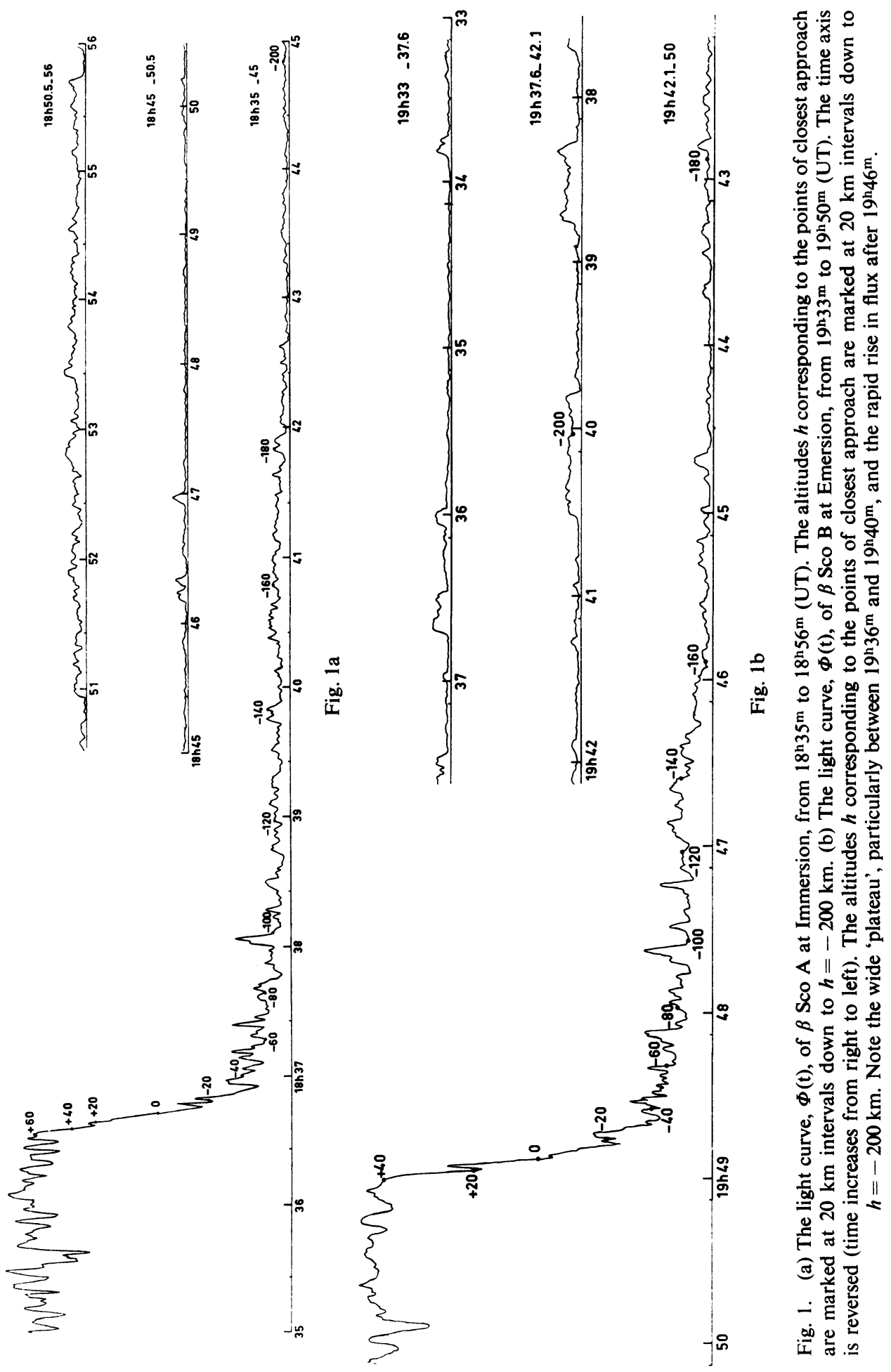




\section{Refractivity Curves}

Since the extinction of the stellar flux is due exclusively to differential refraction in progressively denser layers of the planetary atmosphere, only the major constituents of the atmosphere $\left(\mathrm{H}_{2}\right.$ and $\left.\mathrm{He}\right)$ are important. In what follows, we consider the atmosphere to consist only of these two gases.

We take, as time origin, the time at which the flux from the star has fallen to one

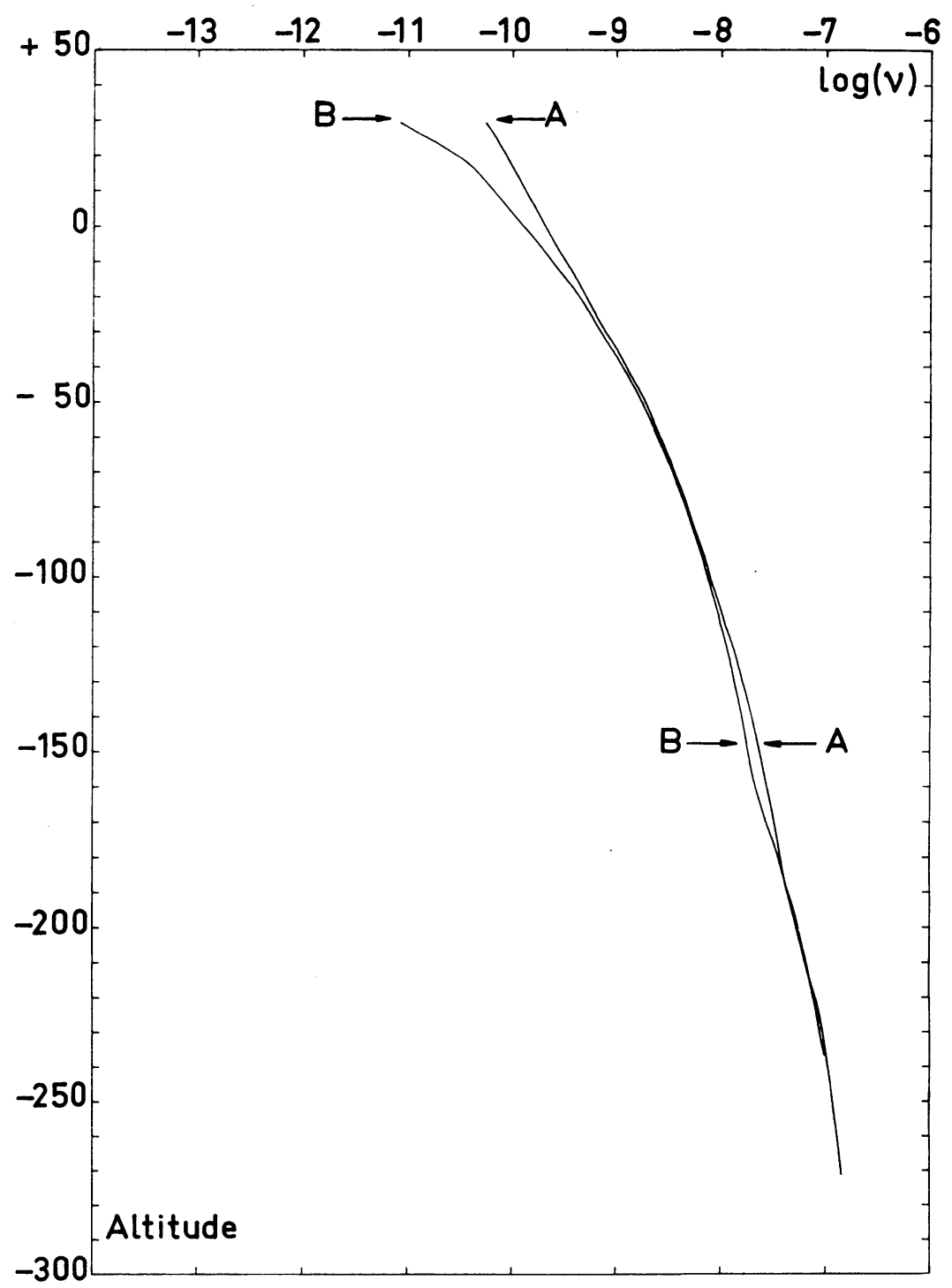

Fig. 2. Refractivity profiles $\log (v(h))$ obtained at Immersion (A) and Emersion (B). Note the divergence between the two profiles above the altitude $h=\mathrm{O}$. 
half of its initial value and, as height origin, the point of closest approach of the light beam at this same time. We see that the curves corresponding to immersion and emersion are very similar for all but the largest values of the flux. This is because the beginning and the end of the occultation are badly defined on the light curves, due to turbulence in the Earth's atmosphere, and the exact form of the light curve at these times is essentially unknown. Consequently, we estimate as $100 \%$ the error in the refractivity at heights greater than $+30 \mathrm{~km}$.

We note in passing that the refractivity curve is not a straight line as the upper atmosphere of Jupiter is not at a constant temperature (Figure 2).

\section{Temperature and Pressure Curves}

From the refractivity profile, it is possible to deduce the temperature and pressure as functions of altitude. These curves are given in Figures $3 a$ and $3 b$, subject to the following conditions:

$$
q=\frac{\mathrm{H}_{2}}{\mathrm{H}_{2}+\mathrm{He}}=0.9 ; \quad h_{0}=-14 \mathrm{~km} ; \quad T\left(h_{0}\right)=150 \mathrm{~K} ;
$$

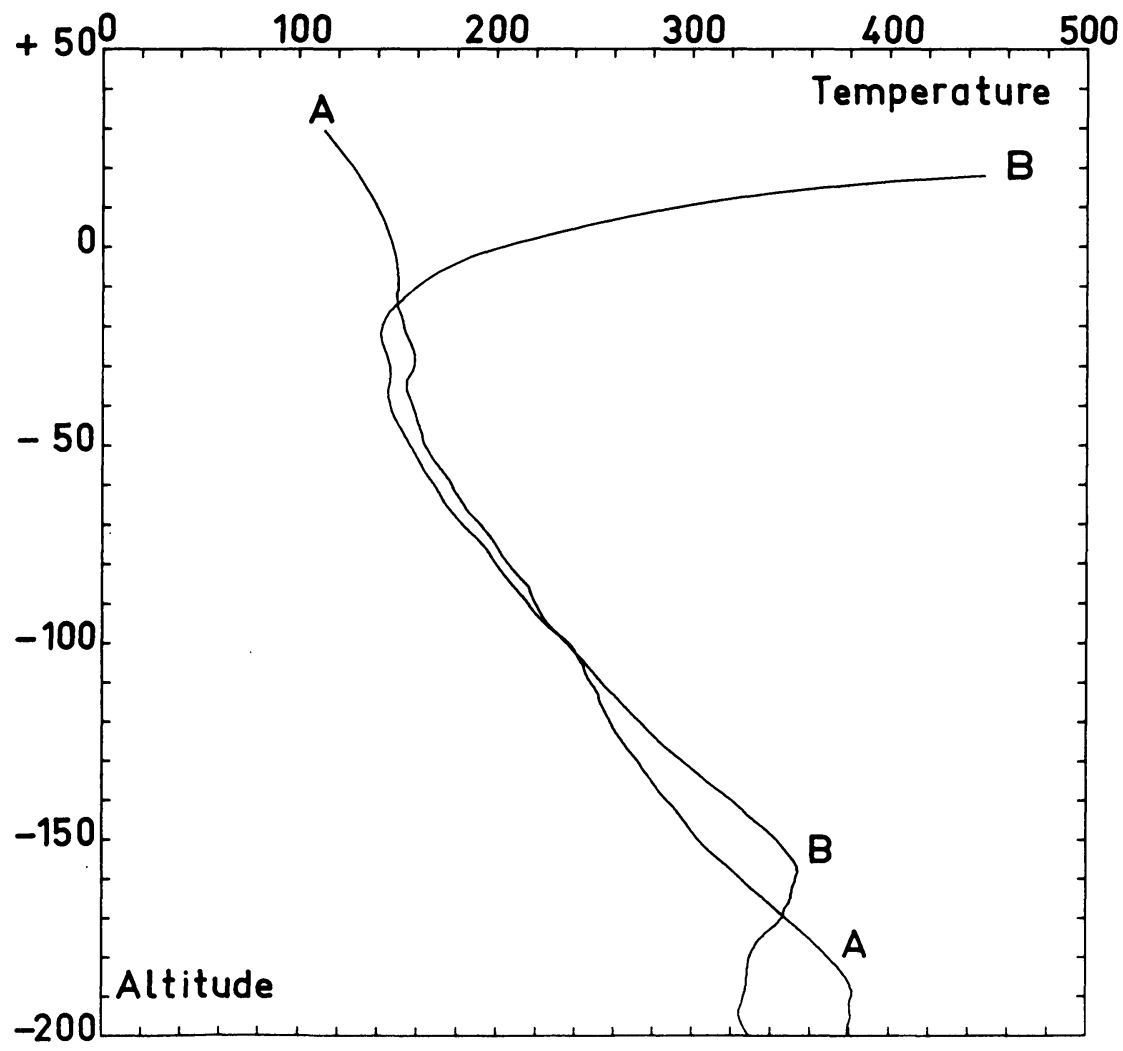

Fig. 3a. 
where $h_{0}$ is the height at which one begins the numerical integrations and $T\left(h_{0}\right)$ is an integration constant whose value is somewhat arbitrary but which can be fixed to within certain reasonable limits. Curve $A$ is for immersion and curve $B$ for emersion. At intermediate altitudes $(-150 \leqslant h \leqslant-50 \mathrm{~km})$, the temperature gradient, $\mathrm{d} T / \mathrm{d} h$, is constant and equal about $-1.5 \mathrm{~K} \mathrm{~km}^{-1}$.

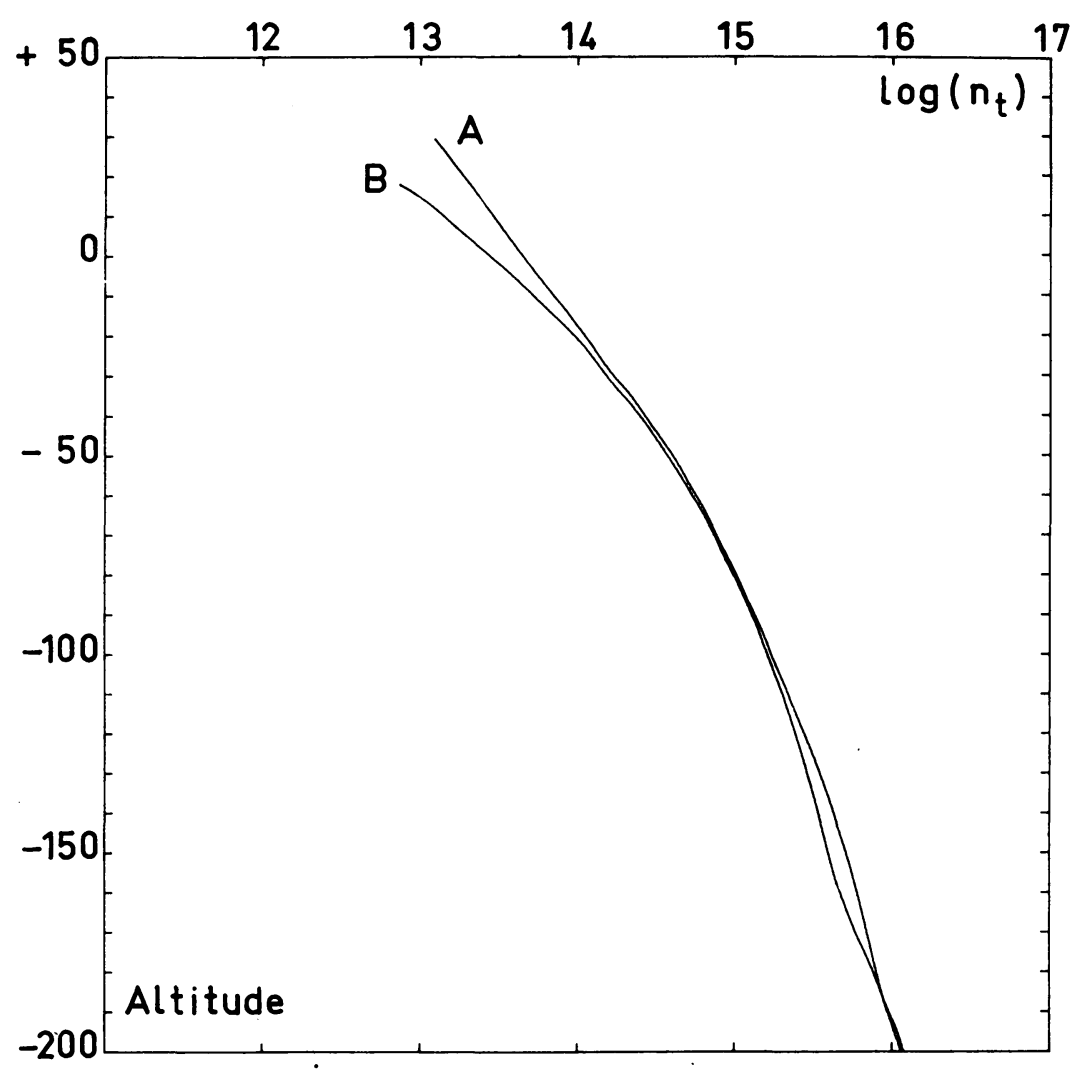

Fig. 3b.

Fig. 3. (a) Typical temperature profiles, $T(h)$, obtained at Immersion (A) and Emersion (B). $q=0.9$; $h_{0}=-14 \mathrm{~km} ; T_{0}=150 \mathrm{~K}$. Note the temperature maximum at $h=-190 \mathrm{~km}(\mathrm{~A})$ and $-160 \mathrm{~km}(\mathrm{~B})$. (b) Typical density profiles $\log \left(n_{t}(h)\right)^{\prime}$, obtained at Immersion (A) and Emersion (B). $q=0.9 ; h_{0}=$ $=-14 \mathrm{~km} ; T_{0}=150 \mathrm{~K}$. Since $v$ and $n_{t}$ are proportional if the atmosphere is wellmixed, i.e. the mixing ratio is altitude independent, Figure $3 \mathrm{~b}$ and Figure 2 are similar.

\section{Importance of the Boundary Conditions}

We now consider the effect on the final results of the choice of $T\left(h_{0}\right)$ (Figure 4). We cannot choose $h_{0}$ too high in the atmosphere, because, as we mentioned above, the corresponding values of the refractivity are very imprecise. Neither can it be too low as the refractivity is then large and this leads to integration problems. We have chosen an optimum value of $h_{0}=-14 \mathrm{~km}$. For this value of $h_{0}$, calculations show 


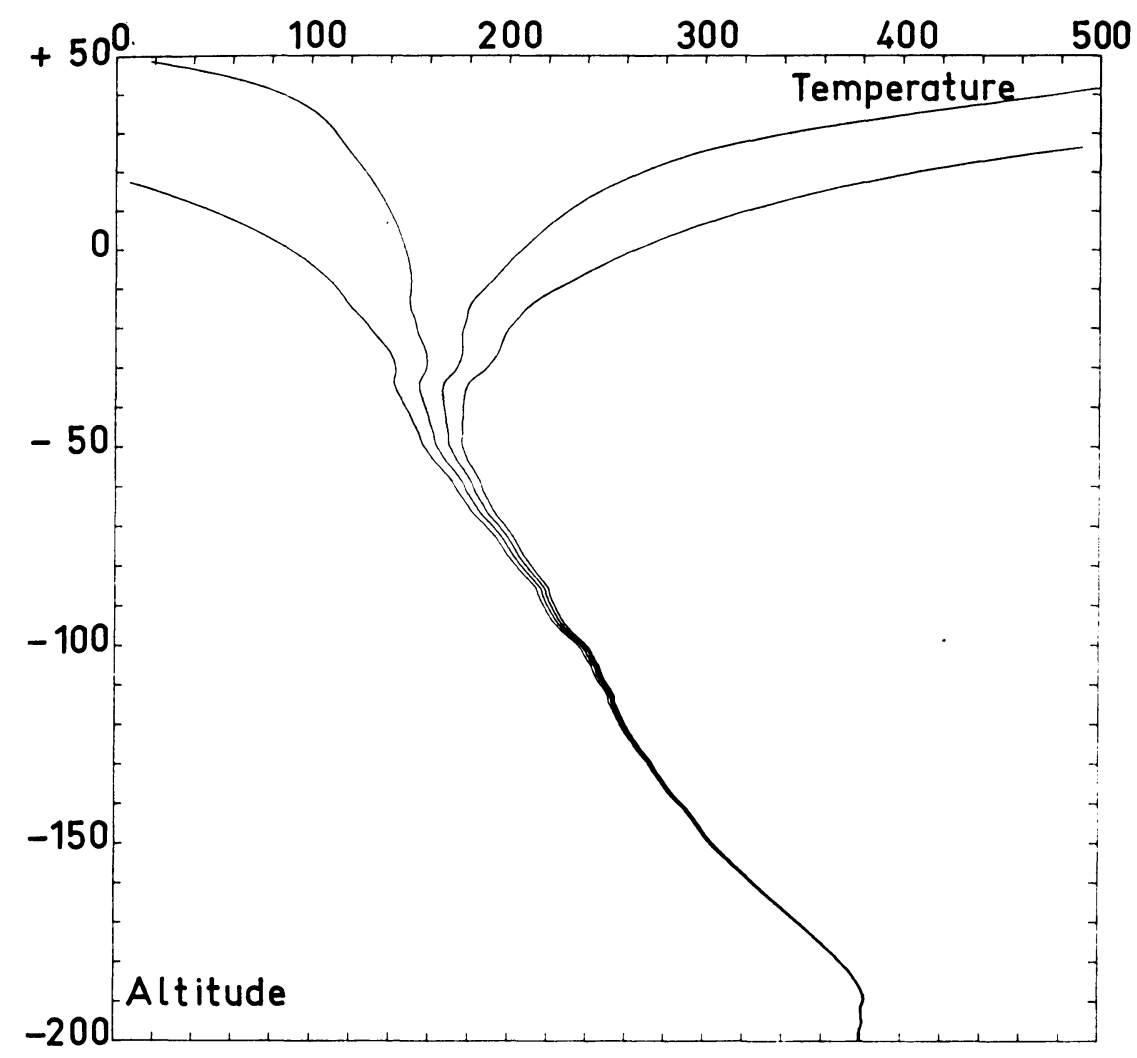

Fig. 4. The influence of the integration parameters $T_{0}$ and $h_{0}$ upon the deduced temperature profile at Immersion. $q=0.9 ; h_{0}=-14 \mathrm{~km} ; T_{0}=120,150,180,210 \mathrm{~K}$. The profile is independent of $T_{0}$ for $h<-100 \mathrm{~km}$.

that the temperature profiles are practically identical for $h \leqslant-50 \mathrm{~km}$ for values of $T\left(h_{0}\right)$ as high as $180 \mathrm{~K}$ or as low as $120 \mathrm{~K}$.

\section{Importance of the Determination of the Initial Stellar Flux}

As we saw above, the determination of the initial and final values of the stellar flux is affected by turbulence in the Earth's atmosphere and this uncertainty introduces an additional error into the profiles of temperature and pressure. We have tried to estimate the importance of this source of error by looking at the effect of a $-1 \%$ (curve C) and $+1 \%$ (curve A) variation in the value of the unocculted stellar flux (Figure 5).

High in the atmosphere, these variations produce large changes in the profiles: it is not possible to draw any meaningful conclusions about conditions in the atmosphere for $h \geqslant+30 \mathrm{~km}$. This height, of $+30 \mathrm{~km}$, is the upper limit of our study. For $h \leqslant+30$ $\mathrm{km}$, however, the effect of the uncertainty in the unocculted stellar flux rapidly becomes negligible. 


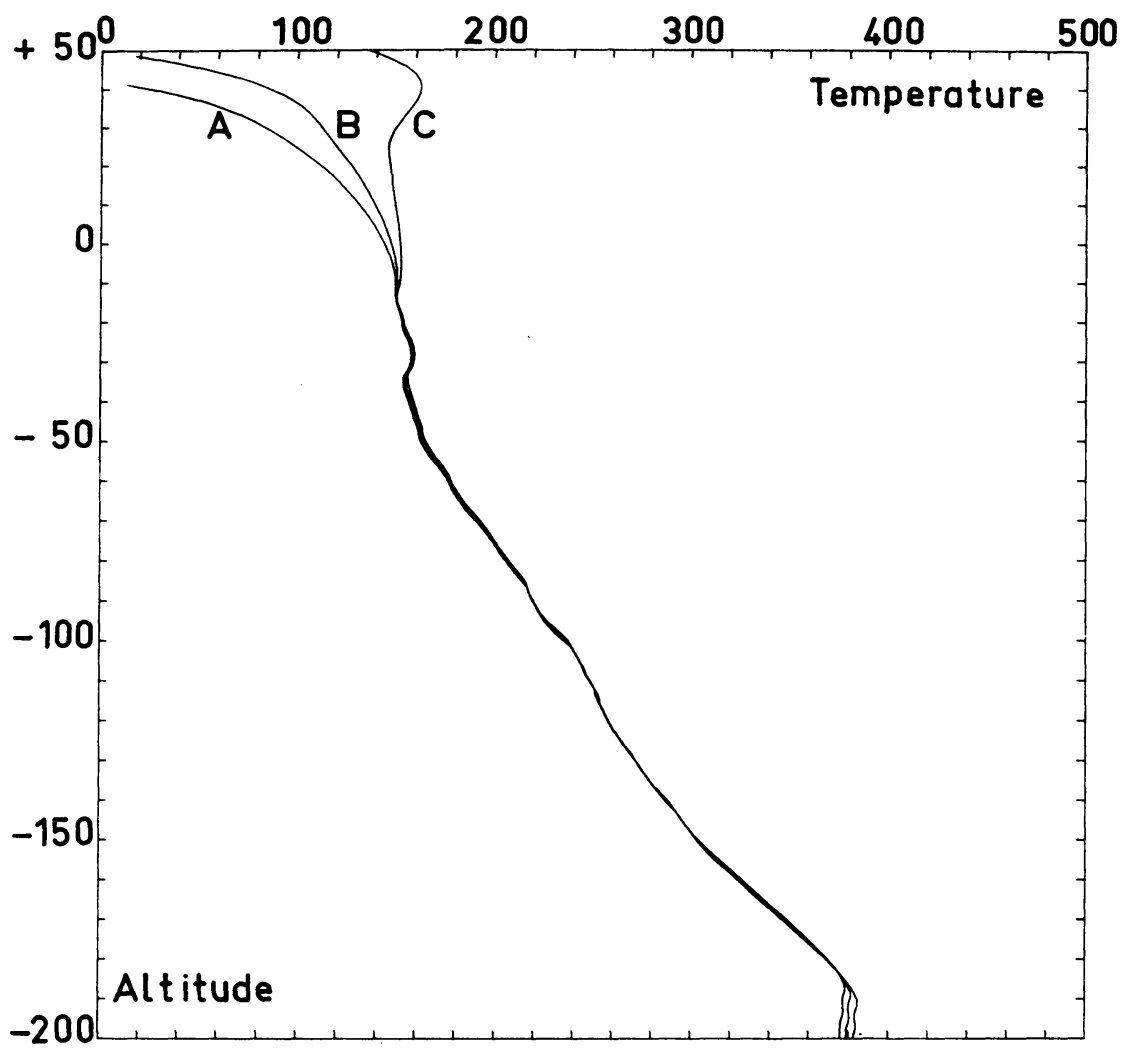

Fig. 5. The influence of a $1 \%$ error in the flux level of $\beta$ Sco A upon the temperature profile at Immersion. A $-\Phi_{0}$ increased by $1 \%$; B - reference curve; C $-\Phi_{0}$ decreased by $1 \% . q=0.9 ; h_{0}=$ $=-14 \mathrm{~km} ; T_{0}=150 \mathrm{~K}$. The profile $T(h)$ is significantly affected only high in the atmosphere $(h>0 \mathrm{~km})$

\section{Importance of the Determination of the Zero Level of the Stellar Flux}

We mentioned in the introduction that the determination of the zero stellar flux level (i.e., the level of background emission from Jupiter) can introduce a significant error. The importance of this error has been estimated by varying the value of this flux level by about $\pm 1 \%$. These changes are unimportant for the high atmospheric layers but very important deep in the atmosphere. A calculation which extended down to $h=280 \mathrm{~km}$ showed that the above variation in the zero flux level results in $T$ being undetermined for $h \leqslant-200 \mathrm{~km}$, whilst in the region $-150 \leqslant h \leqslant-50 \mathrm{~km}$ the temperature and especially $\mathrm{d} T / \mathrm{d} h$ are perfectly defined. We have, therefore, taken $h=-200 \mathrm{~km}$ as the lower limit of our investigation (Figure 6).

\section{Importance of Helium}

Finally, we consider the variation of the calculated profiles with the relative abun- 


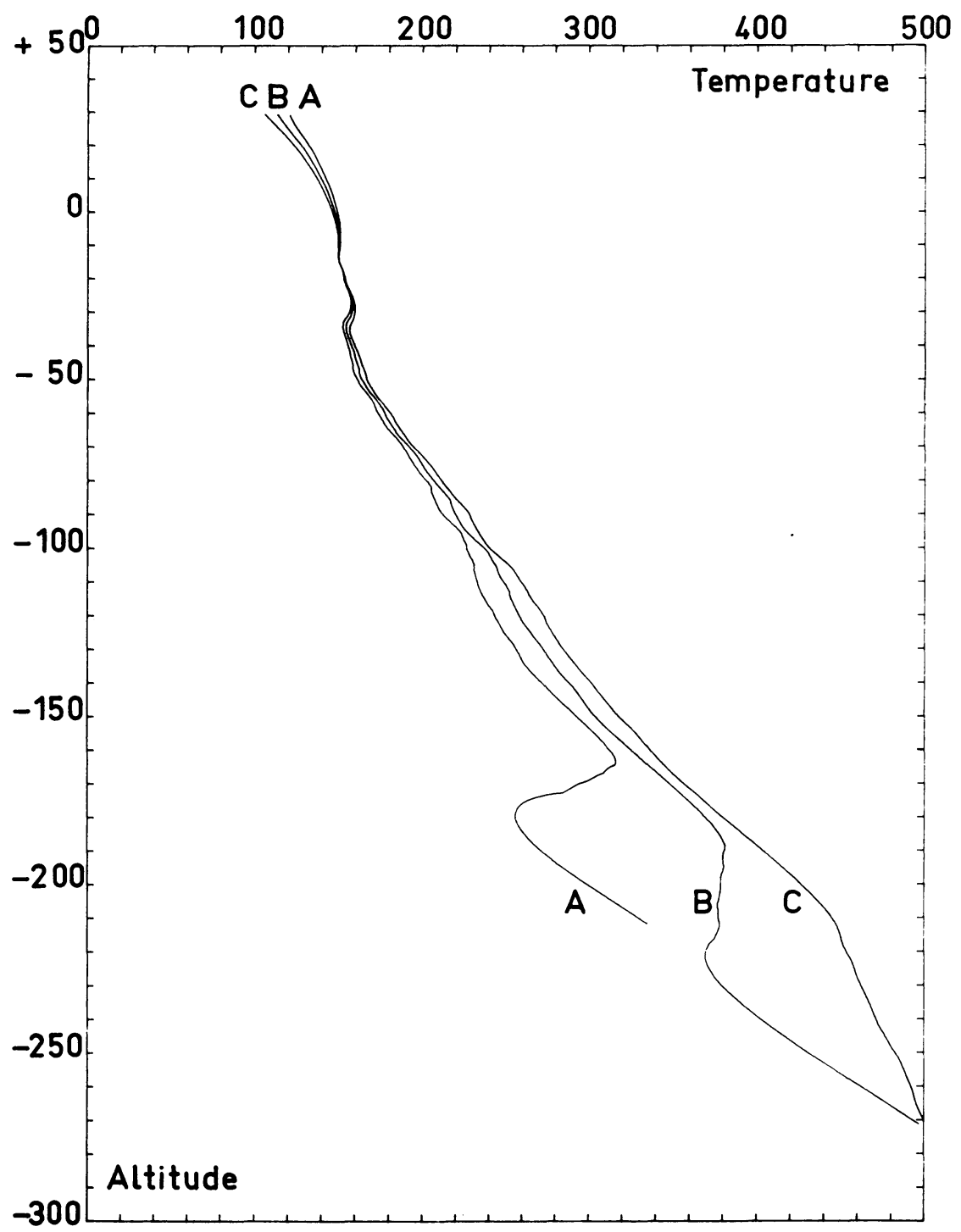

Fig. 6. The influence of an $1 \%$ error in the Jovian background light upon the temperature profile at Immersion. A - background light level increased by $1 \% ; \mathrm{B}$-reference curve; $\mathrm{C}$ - background light level decreased by $1 \% . q=0.9 ; h_{0}=-14 \mathrm{~km} ; T_{0}=150 \mathrm{~K}$. The profile $T(h)$ is appreciably affected deep in the atmosphere $(h<-100 \mathrm{~km})$. Note the displacement of the temperature maximum.

dance of helium (Figure 7). Calculations were carried out for $q=1,0.9,0.8$ and 0.7 .

Between -50 and $-150 \mathrm{~km}$, the gradient of the temperature profile remains independent of $h$ but increases by a factor 1.3 for an increase in the helium abundance from $0 \%$ to $30 \%$. Such a result was to be expected as the introduction of helium is known to lead to an increase in temperature for a given value of the refractivity. Our measurements would seem to exclude the possibility that helium is the major con- 
RESULTS BY THE BETA SCORPII OCCULTATION BY JUPITER ON MAY 13, 1971

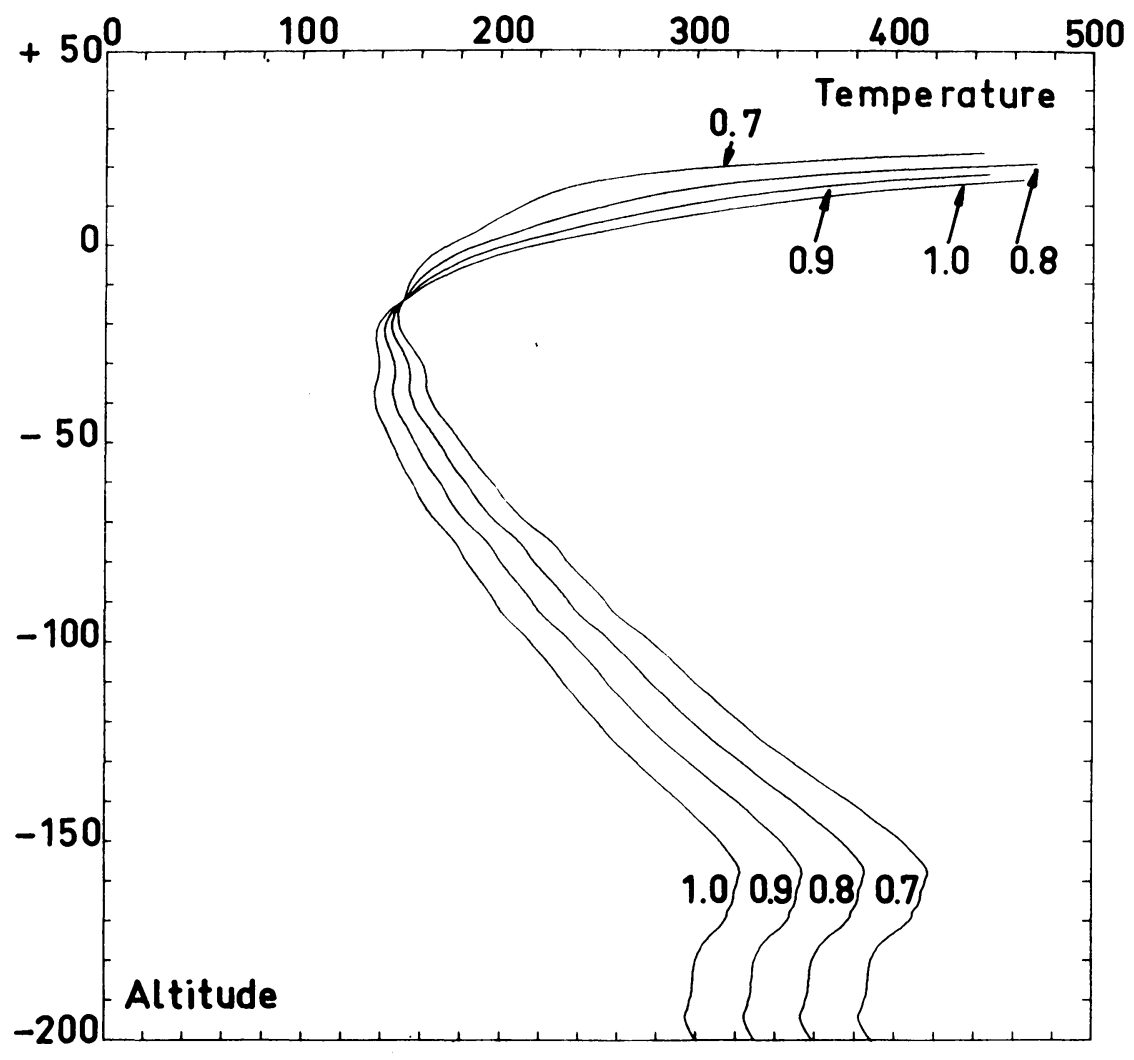

Fig. 7. Influence of the mixing ratio, $q=\mathrm{H}_{2} / \mathrm{H}_{2}+\mathrm{He}$, at Emersion. $q=1.00$ (A), 0.90 (B), 0.80 (C), 0.70 (D); $h_{0}=-14 \mathrm{~km} ; T_{0}=150 \mathrm{~K}$.

stituent of Jupiter's atmosphere as in this case, the calculated temperatures for $-200 \leqslant h \leqslant-150 \mathrm{~km}$ are unreasonably high.

\section{Conclusions}

(1) The $\mathrm{H}_{2} / \mathrm{He}$ abundance ratio cannot be precisely determined but is probably such that $q>0.7$. An in situ measurement of the temperature in the atmosphere of Jupiter would clarify this point.

(2) The temperature profile is well determined as a function of $q$ for $-150 \leqslant h \leqslant-$ $-50 \mathrm{~km}$ and the gradient is $\mathrm{d} T / \mathrm{d} h \simeq-1.5 \mathrm{~K} \mathrm{~km}^{-1}$ (slightly dependent on $q$ ).

(3) The temperature reaches a maximum value, $T_{\max } \geqslant 300 \mathrm{~K}$, between -160 and $-190 \mathrm{~km}$.

(4) No information on the thermosphere can be derived as the light curve for this region is too noise dependent.

Note of the editors. A more extensive description of this observation can be found in: Vapillon, L., Combes, M., and Lecacheux, I.: 1973, Astron. Astrophys. 29, 135. 